\title{
Survey Catalogues of Kiso Ultraviolet-Excess Galaxies
}

\author{
Nagako Miyauchi-Isobe \\ National Astronomical Observatory, Mitaka, Tokyo 181, Japan \\ Bunshiro Takase \\ Kokugakuin University, Shibuya-ku, Tokyo 150, Japan
}

\section{Sadanori Okamura}

Department of Astronomy, School of Science, University of Tokyo, Tokyo 113, Japan

\begin{abstract}
Our survey for KUGs (Kiso Ultraviolet-excess Galaxies) is based on the multi-colour method applied to photographic plates taken with the Kiso Schmidt telescope. In total, 8,968 KUGs were detected on 170 plates covering some 5,100 square degrees. They are listed in 17 separate catalogues, where morphological type and UV-excess degree are given for each object together with position, size, and magnitude. After the appropriate editing of multiple entries of the same object detected on different plates, we produced a merged catalogue where $8,162 \mathrm{KUGs}$ were arranged in order of right ascension. The accuracy of the position of KUGs was examined using the data of $806 \mathrm{KUGs}$ whose positions were measured on different plates.
\end{abstract}

\section{Introduction}

A survey for KUGs (Kiso Ultraviolet-excess Galaxies) has been carried out with the Kiso $100 \mathrm{~cm}$ Schmidt telescope since the late $1970 \mathrm{~s}$, when a series of catalogues of Markarian galaxies was being sucessively published on the basis of objective prism plates taken with the Byurakan $100 \mathrm{~cm}$ Schmidt telescope.

We used UGR three-colour (later often UR two-colour) photographs instead of the Byurakan spectral method. However, the detection criterion for ultraviolet-excess galaxies is quite similar between Byurakan and Kiso. Markarian and collaborators compared the relative strength of the two portions of the objective prism spectrum, the violet-blue side and the yellow-red side, separated by the so-called green dip, while we compared the U, (G), and R images, which were exposed adjacently on the same plate. Our exposure times were adjusted so that the three colour images of an A0 star appeared equally bright on the plate.

Our method is based on direct images and it has a few advantages over the Byurakan method, at the cost of spectral information. Our limiting magnitude is fainter by at least 1 magnitude and detailed morphological features including 


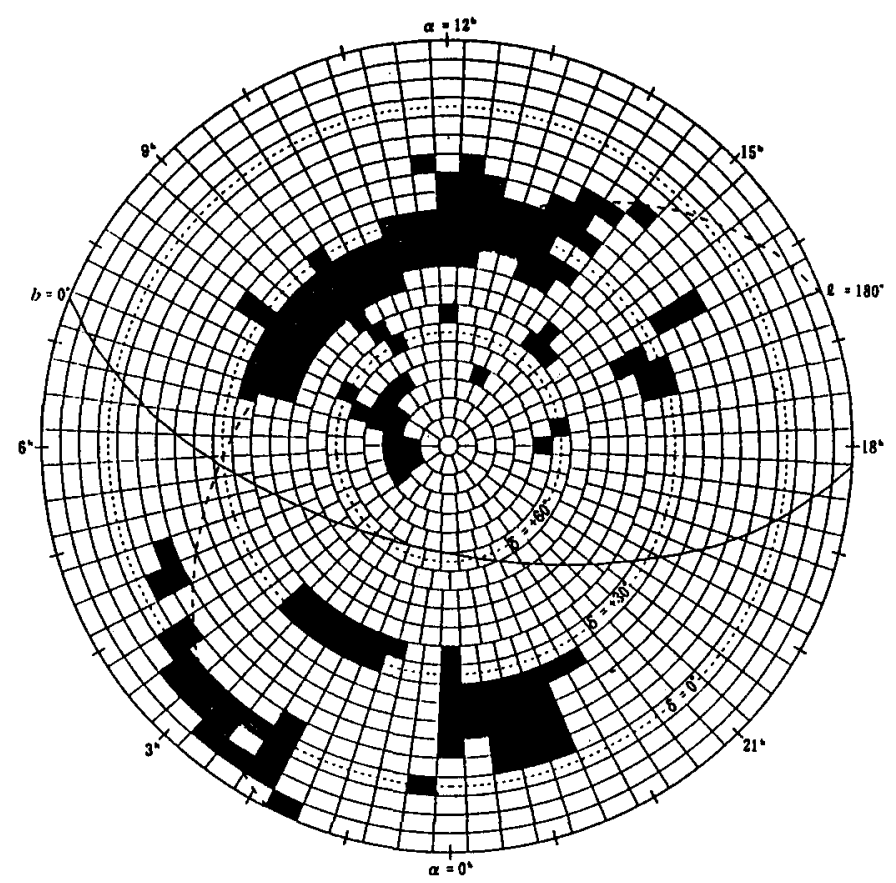

Figure 1. Distribution of the surveyed areas for KUG

the distribution of blue regions in a galaxy are visible on the plate. Our multicolour method owes much to a unique mechanical feature of the Kiso Schmidt; any of three filters that are preloaded in filter holders in the telescope tube can be selected remotely from the control desk without interrupting the telescope guiding.

\section{Results of the Survey}

Our initial survey plan was to cover the Kiso celestial areas located along the great circle of galactic longitude $l=180^{\circ}$ together with those which include several Markarian galaxies. However, this plan has not been well fulfilled during the course of the actual survey observation. The distribution of the areas surveyed so far is fairly scattered as seen in Fig. 1.

In total, 8,968 KUGs have been detected in 170 areas. Adjacent areas are overlapped. Those KUGs that are located in the overlapped regions were detected on different plates independently and entered the catalogues repeatedly. After the deletion of multiple entries of the same object, the net number of KUGs results in 8,162 . One area covers about 30 square degrees, so the total surveyed area amounts to some 5,100 square degrees. The surface number density is thus 

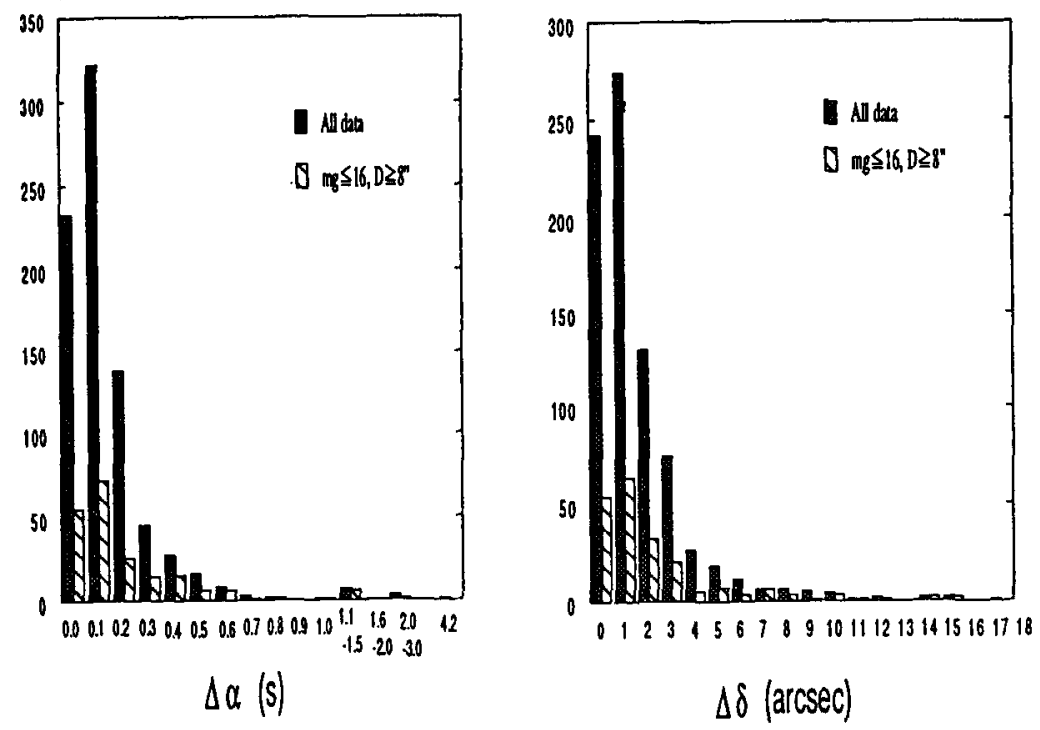

Figure 2. Histograms of position differences measured on different plates. Shaded and hatched parts are for the total and bright samples, respectively.

found to be $1.8 \mathrm{KUGs}$ per square degree, which is nearly 20 times as high as that of Markarian galaxies (0.1 per square degree).

The limiting magnitudes of the survey range from 17 to 18.5 photographic magnitude, depending on observing conditions and plate qualities. It should be noted, therefore, that the survey is not very homogeneous over the whole surveyed area.

\section{Catalogues of KUGs}

During the past ten years, 17 catalogues of KUGs were published sequentially (Takase and Miyauchi-Isobe 1984-1993a: KUGC I-XVII). Each catalogue includes KUGs detected in ten areas and lists coordinates, morphological type, size, magnitude, and UV-excess degree, together with other names, if any. The finding chart for every KUG is also given as an appendix of the catalogues.

Efforts were made to give consistent data for objects that were detected repeatedly on different plates. KUG name, type, size, magnitude, and UVexcess degree were reconciled whenever the same object appeared again in the later catalogues (see errata tables attached to KUGC II through XVIII).

Recently, we produced a new catalogue of KUGs (Takase \& Miyauchi-Isobe 1993b: KUGC XVIII, see Table 1) by merging the original 17 catalogues together. The multiple entries were deleted and all 8,162 KUGs detected so far are arranged in order of right ascension. Each KUG in the merged catalogue 

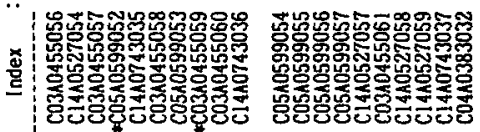

苛 (1)

垔

i........ is

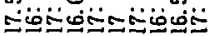

-n-m-6nN-N

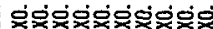

i. \$00000000

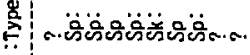

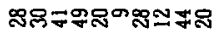

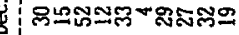

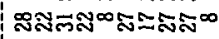

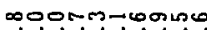

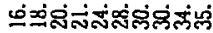

3

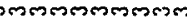

(1)

(1)

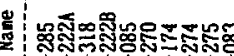

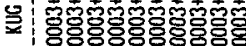

$\because$.

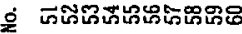
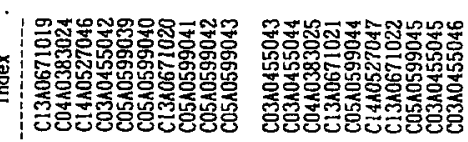

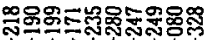

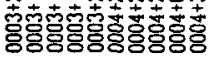

is 0 ... in

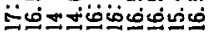

NNMTNNANANO

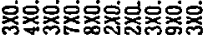

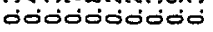

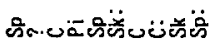

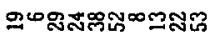

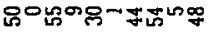

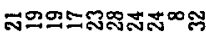

amnistor-torm

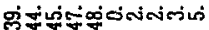

menmmatar

0000000000

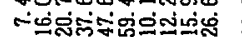

-ratarisurion

0000000000

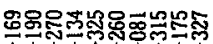

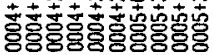

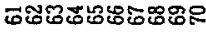

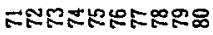

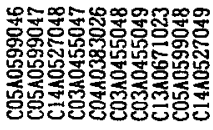

|

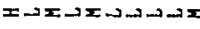

is . n 0 ro...

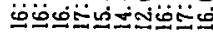

- inis orinis

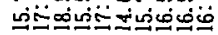

No-mmrnmo

NNTNM-NANM

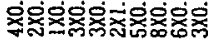

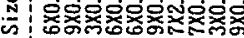

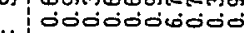

indodidión

is

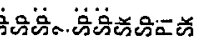

莽ง

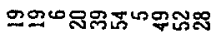

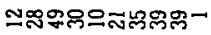

\&

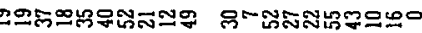

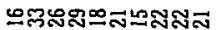

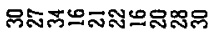

minoo-rnu-co

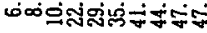

$\infty-N 000 m 000$

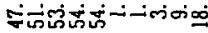

$00000 \pi-\cdots+\cdots$

$x=x \rightarrow x-x+x x$

ismois is on

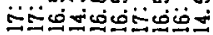

NMN-NMNN-U

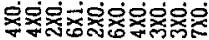

soditiojosos

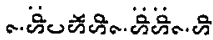

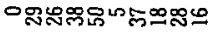

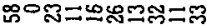

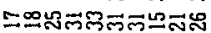

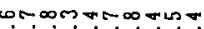

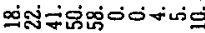

7-n-mannana

0000000000

0000000000

0000000000

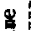

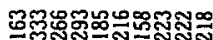

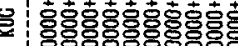

$\dot{0}$

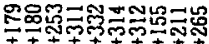

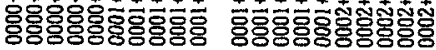

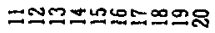

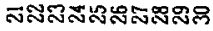
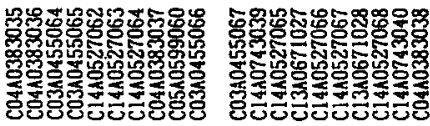

ה

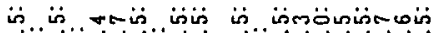

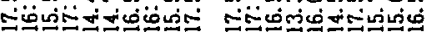

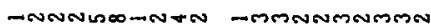

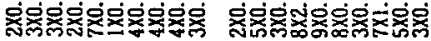

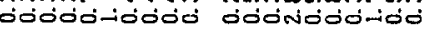

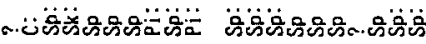

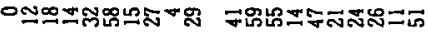

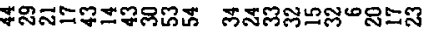

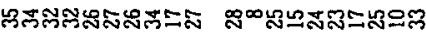
o-oisin-wiso monamotumco

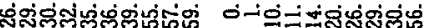

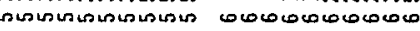
0000000000 0000000000

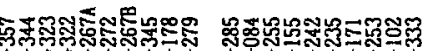

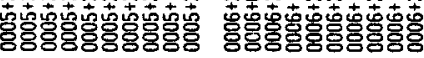

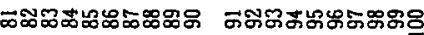
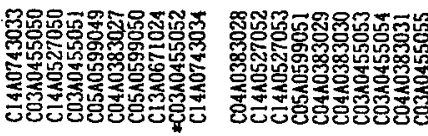

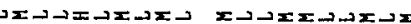
isin in is is

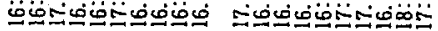

MMMNM-MN-A -M-NNד-MNN

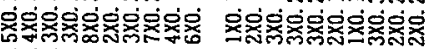

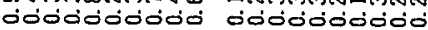

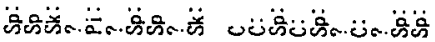

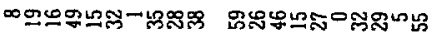

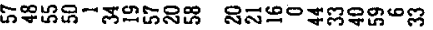

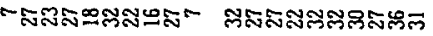

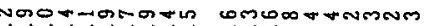

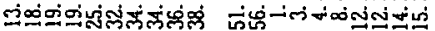
NNNNNNNNNN NNmmmmmmmm 00000000000000000000

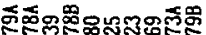

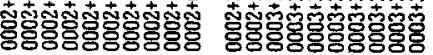

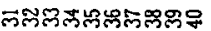


has an index which enables us to identify its location in the 17 catalogues. An asterisk (*) is attached to the index for KUGs with multiple entries. The index for those KUGs corresponds to their first entry, and the indices for later entries are given in the last column of Notes. General descriptions of the survey and some statistics are also given in the merged catalogue.

The morphological types in the catalogues are given based on the classification scheme presented by Takase et al. (1983). When the type is not so certain, a colon (:) is attached to the type designation. The meaning of each type designation is as follows;

Ic: Irregular with clumpy HII regions

Ig: Irregular with a conspicuous giant HII region

Pi: Pair of interacting components

Pd: Pair of detached components

Sk: Spiral with knots of HII regions along arms

Sp: Spiral with peculiar bar and/or nucleus

C: Compact

?: Unclassifiable

The UV-excess degree is estimated from the appearance of the KUG on the multi-colour plate. The letters $\mathrm{H}, \mathrm{M}$, and $\mathrm{L}$ mean high, medium, and low, respectively. Explanations of this UV-excess degree can be found in Takase et al. (1983). For the KUGs with multiple entries, right ascension and declination given in the merged catalogue are averaged values. The difference among multiple entries is typically less than a few arcsec (see the next section).

\section{Positional Accuracy}

In the course of preparing the merged catalogue, the accuracy of the catalogued position was examined using the data for 806 KUGs which were detected more than once on different plates.

Positions of KUGs were measured on the plate with the XY measuring machine at the Kiso Observatory. The centre of each KUG was defined by visual inspection of the image on the monitor screen of the machine. When the nucleus was visible, the center was unambiguously defined. However, quite a few KUGs had fairly irregular shapes and the definition of the center for those KUGs, in particular, for large bright ones, was subject to some uncertainty.

The measured $(x, y)$ positions were converted to the equatorial coordinates using the standard coordinate method. About 25 position standard stars were used for each plate. Those stars were taken from either AGK3 or SAO catalogue (see Noguchi et al. 1980). The nominal accuracy of the catalogue position at the epoch 1985.0 are 0.4 arcsec and 0.9 arcsec for AGK3 and SAO, respectively. The typical positional error for a star measured on a Kiso Schmidt plate was estimated to be 0.17 arcsec on the basis of the analysis of 9 plates (Nakamura and Sekiguchi 1993). This value includes the bisection error and the random and systematic errors due to plate modelling. The error in the catalogue position is not included, however. The corresponding error would be slightly larger for our 
plates because of the longer exposure time. However, the catalogue error is still the dominant component in the position error of a stellar object in our case.

We examined the position error of non-stellar KUGs. The 806 KUGs were divided into bright and faint samples. The bright sample includes KUGs with diameter larger than 0.8 arcsec and also with magnitude brighter than 16 photographic magnitude. Fig. 2 shows the histogram of their position differences measured on different plates. The shaded and the hatched parts are for the total and the bright samples, respectively. The data for AGK3 and SAO are not discriminated here.

Twelve KUGs which show large position differences $\left(\Delta \alpha>0.9^{s}, \Delta \delta>9^{\prime \prime}\right)$ all belong to the bright sample. This indicates the difficulty in defining the center of large bright KUGs noted above. Except for these $12 \mathrm{KUGs}$, the rms position difference is $\Delta \alpha \sim 0.1^{s}$ and $\Delta \delta \sim 2^{\prime \prime}$. These are roughly 2-3 times the estimated error for stellar objects. These numbers may be regarded as the typical position accuracy of the KUG catalogues although the accuracy for faint KUGs is slightly better as seen in Fig. 2.

\section{Acknowledgements}

We would like to thank the staff of the Kiso Observatory for continuous help throughout this project, and M. Hamabe and N. Takato for preparing the figures. NMI appreciates the travel fund from Japan Society for the Promotion of Science.

\section{References}

Nakamura T. \& Sekiguchi M., 1993, PASJ, 45, 119

Noguchi T., Maehara H. \& Kondo M., 1980, Annals Tokyo Astron. Obs. 2nd Ser., 18,55

Takase B.\& Miyauchi-Isobe N., 1984, Annals Tokyo Astron. Obs. 2nd Ser., 19, 595 (KUGC I)

a, Annals Tokyo Astron. Obs. 2nd Ser., 20, 237 (KUGC II)

Takase B. \& Miyauchi-Isobe N., 1985b, Annals Tokyo Astron. Obs. 2nd Ser., 20,335 (KUGC III)

Takase B. \& Miyauchi-Isobe N., 1986a, Annals Tokyo Astron. Obs. 2nd Ser., 21,127 (KUGC IV)

Takase B. \& Miyauchi-Isobe N., 1986b, Annals Tokyo Astron. Obs. 2nd Ser., 21,181 (KUGC V)

Takase B. \& Miyauchi-Isobe N., 1987a, Annals Tokyo Astron. Obs. 2nd Ser., 21,251 (KUGC VI)

Takase B. \& Miyauchi-Isobe N., 1987b, Annals Tokyo Astron. Obs. 2nd Ser., 21,363 (KUGC VII)

Takase B. \& Miyauchi-Isobe N., 1988, Annals Tokyo Astron. Obs. 2nd Ser., 22, 41 (KUGC VIII)

Takase B. \& Miyauchi-Isobe N., 1989a, Publ. National Astron. Obs., 1, 11 (KUGC IX) 
Takase B. \& Miyauchi-Isobe N., 1989b, Publ. National Astron. Obs., 1, 97 (KUGC X)

Takase B. \& Miyauchi-Isobe N., 1990, Publ. National Astron. Obs., 1, 181 (KUGC XI)

Takase B. \& Miyauchi-Isobe N., 1991a, Publ. National Astron. Obs., 2, 7 (KUGC XII)

Takase B. \& Miyauchi-Isobe N., 1991b, Publ. National Astron. Obs., 2, 37 (KUGC XIII)

Takase B. \& Miyauchi-Isobe N., 1991c, Publ. National Astron. Obs., 2, 239 (KUGC XIV)

Takase B. \& Miyauchi-Isobe N., 1992a, Publ. National Astron. Obs., 2, 399 (KUGC XV)

Takase B. \& Miyauchi-Isobe N., 1992b, Publ. National Astron. Obs., 2, 573 (KUGC XVI)

Takase B. \& Miyauchi-Isobe N., 1993a, Publ. National Astron. Obs., 3, 21 (KUGC XVII)

Takase B. \& Miyauchi-Isobe N., 1993b, Publ. National Astron. Obs., 3,169 (KUGC XVIII)

Takase B., Noguchi T. \& Maehara H., 1983, Annals Tokyo Astron. Obs. 2nd Ser., 19,440 